\title{
The working of the IGRF 2000 Task Force
}

\author{
F. J. Lowes \\ Physics Department, University of Newcastle, Newcastle upon Tyne, NE1 7RU, U.K. \\ (Received February 14, 2000; Revised May 24, 2000; Accepted May 24, 2000)
}

\begin{abstract}
At its meeting in July 1999, the IAGA Working Group for Analysis of the Global and Regional Geomagnetic Field and Secular Variation (WG V-8) felt unable to decide on a main-field model to use for IGRF 2000. It therefore set up a small Task Force with the remit to produce a model by the end of the year. This paper is the Chairman's report of the working of that Task Force, outlining the various stages involved, and giving the background to the various decisions. He also makes some retrospective personal comments.
\end{abstract}

\section{Introduction}

As was usual, candidate models for IGRF 2000 were submitted some months before the meeting of IAGA at Birmingham in July 1999, and assessments of these models were presented at the meeting of IAGA Working Group V-8. The intention was that, as usual, the Working Group would decide on what set of coefficients (usually some average) to adopt. However on this occasion none of the submitted main-field models appeared very good; in fact on some of the tests the IGRF 1995 updated to 2000 using the 7th Generation 1995-2000 secular variation appeared better! (See Macmillan, 2000 and Mandea and Langlais, 2000.) It was hoped that vector data from the magnetometer satellite Ørsted would soon become available, so it was decided to defer a decision. A small Task Force was set up, consisting of Frank Lowes (University of Newcastle, UK) Chairman, Vadim Golovkov (IZMIRAN, Russia), Susan Macmillan (BGS, UK), Mioara Mandea (IPGP, France), and Terry Sabaka (GSFC, USA), with the remit to produce an IGRF 2000 model in time for it to be published before the end of the year. The Working Group also decided that the fall-back position was to use the updated IGRF 1995.

This paper is a brief report by the Chairman on the working of the Task Force, together with some personal comments.

\section{Initial Meeting}

The Task Force held a meeting during the IAGA Assembly. After discussion with Susan McLean (NOAA National Geophysical Data Center) it was decided that the model would be produced by 1 December 1999 . This would minimize the impact on the many users of the IGRF, as IGRF 1995 expired on 31 December 1999.

After briefing by Nils Olsen (Ørsted Project, International Coordinator of Internal Field Sciences and Modelling) on the current status of, and apparent problems with, Ørsted data, the following procedure and timetable were agreed:

Copy right (C) The Society of Geomagnetism and Earth, Planetary and Space Sciences (SGEPSS); The Seismological Society of Japan; The Volcanological Society of Japan; The Geodetic Society of Japan; The Japanese Society for Planetary Sciences.
Stage 1 Model Using only Ørsted $(X, Y, Z)$ and $F$ data, Olsen and Sabaka would produce a model by 1 October.

Stage 1 Assessment The Stage 1 model would be assessed by comparison with a Stage 1 set of recent surface data, together with a set of Ørsted $F$ data not used in the modelling. The assessment might be influenced by whether or not the current problems with the Ørsted vector data had been resolved.

If acceptable the Stage 1 Model would be adopted. If not acceptable proceed to Stage 2.

Stage 2 Model This would use only $F$ data from Ørsted, together with a Stage 2 set of surface data (which might involve some augmentation of the Stage 1 set of surface data), and would be produced by 1 November.

Stage 2 Assessment A decision on the acceptability of the Stage 2 model would be made before 1 December.

If acceptable the Stage 2 Model would be adopted. If not acceptable adopt the WG V-8 fall-back position of the 7th Generation IGRF, i.e. IGRF 1995 updated to 2000.0 using the current IGRF secular variation.

The meeting also decided on the sampling criterion for the Ørsted data (every 20 seconds along track), together with $\sin \theta$ weighting (so as to approximate to uniform surface coverage); the vector data were to be limited to $\leq 50^{\circ}$ geomagnetic latitude. Also, (almost) all data and/or models, both surface and satellite, would be extrapolated to epoch 2000.0 using the current IGRF 1995 secular variation. (Although not stated explicitly, it was expected that the resulting candidate model(s) would be accompanied — as had the other candidate models - by a report describing the main features of its derivation.) In addition, various data-gathering jobs were allocated.

After this initial meeting, the Task Force work was almost all done by e-mail (I logged 130 messages!), though I was able to visit Terry Sabaka at GSFC. The modelling was done by Nils Olsen and Terry Sabaka. Tatiana Bondar (IZMIRAN) and Benoit Langlais (IPGP) helped in the model assessment. 


\section{Stage 1 Model}

The Ørsted vector magnetometer was itself working to within specification. Unfortunately there were initially several problems with the spacecraft stabilization, and hence determination of its orientation using the star camera, so that orientation was available only for limited periods, particularly for the earlier months. Also, when the magnetometer data were rotated to a ground-based reference frame, using those star camera observations that were available, there were several worrying features in the resultant vector data. It was only on 27 September that it was realized by the Ørsted team that (most of) the problem was due to a timing difference between the magnetometer and the star camera. Even so, using the corrected data a model was produced by 1 October, and a report on the method used (6 pages plus diagrams) by 12 October.

The parent model Ørsted(9/99) was taken to $n=13$ for internal field; and to $n=1$ for external field; the $n=1$ coefficients (except for $g_{1}^{1}, h_{1}^{1}$ ) were allowed to vary linearly with Dst. The modelling included the estimation of the relative orientation of the magnetometer and star camera. The model was produced using only the data from 6 quiet days in May (having 2215 vector and $7328 F$ observations), so as to leave other data for comparison. The IGRF candidate, locally called IGRF2000a, consisted of the constant internal terms of Ørsted(9/99) truncated to $n \leq 10$.

\section{Assessment of Stage 1 Model \\ 4.1 Data preparation}

All the recent ground data available to BGS and IPGP were carefully assessed, and when accepted brought up to epoch. Observatory data

Where monthly means were available these were checked, and extrapolated to 2000.0 using exponential smoothing (as explained in Langlais and Mandea, 2000). Where only annual means were available, the last one was extrapolated using the IGRF secular variation (174 observatories in total).

Where a value was available for 1980.0 , this was compared with a $n \leq 131980.0$ model (based on MAGSAT data) to estimate the observatory bias (the apparent crustal field at that site). Bias estimates were established for 150 of the 174 observatories. These bias estimates were subtracted from the 2000.0 values before the assessment.

\section{Repeat stations}

There were good data from 712 recent occupations of repeat stations.

\section{Marine data}

The marine $F$ data from 1995 to 1998 already selected and updated to 1997.5 by Langlais and Mandea (2000) were extrapolated to 2000.0 using the IGRF secular variation (139 points).

\section{Ørsted $\boldsymbol{F}$ data}

These were night-time data selected for quiet periods (not used in the modelling) from March to August, and extrapolated to 2000.0 using the IGRF secular variation (upwards of 500000 points).

\subsection{Results}

The numerical results were very little different from those reported in Lowes et al. (2000) for the assessment of the later IGRF2000c Ørsted model, so will not be presented here. An independent look at observatory annual means by Bondar and Golovkov gave similar results.

There was considerable discussion about various aspects of the modelling, and of the results of the assessment. But the eventual decision was that this IGRF2000a model was good enough as an IGRF model, so that there was no need to go on to the original proposed Stage 2 modelling. However the modellers were sure that, given a little more time, they could use more Ørsted data to give a better model, and the Task Force agreed on 15 October to give them until 1 November to do this.

\section{Second Ørsted Vector Model}

This model, Ørsted(10c/99), was produced on 1 November, together with another 3 pages of report, plus extensive diagrams. It used essentially the same technique as the Stage 1 model, except that the data were taken every 30 seconds rather than every 20 seconds, and that a smoothed version of the star-camera data was used. The data were from all quiet 3-hour periods March to September (satisfying a rather more rigorous criterion than used for the earlier model), so that there were 20,600 $F$ data and 4054 vector data. The IGRF 1995 secular variation was (effectively) imposed. For full details see Olsen et al. (2000). The IGRF candidate model, called IGRF2000c, consisted of the constant internal terms of Ørsted(10c/99) truncated to $n \leq 10$.

\section{Assessment of Second Ørsted Vector Model}

The surface data-base was slightly updated and expanded from that of the first assessment, and many more Ørsted $F$ data (not used in the modelling), from March to September, were used. An account of the data selection, and the results of the numerical assessment, are given in Lowes et al. (2000). The rms residuals obtained for the repeat station $(X, Y, Z, F)$, the observatory $(X, Y, Z)$ without crustal correction, and the marine $F$, were consistent with what we know of the magnitude of the crustal field; see e.g. Langel et al. (1989). The figures for the observatories incorporating the crustal correction were, as expected, a lot smaller. Our $(X, Y, Z)$ figures of about $(50,60,110) \mathrm{nT}$ were still quite a bit larger than the $(20,20,40)$ obtained by Sabaka et al. (1997, figure 16), but it must be remembered that they were using a crustal bias and secular variation determined in the same analysis. Also they were using their full model out to $n=13$; the $n=11-13$ harmonics themselves contribute about $(20,20,25) \mathrm{nT}$.

Ørsted $F$ data from various sets of quiet 3-hour periods were used. For the quietest data set, the mean deviation was about $5 \mathrm{nT}$, and the standard deviation about $15 \mathrm{nT}$. Again there was the complication that the comparison was with the purely internal, $D s t=0$, truncated $n \leq 10$, model. Sabaka (private communication) had shown that while the earlier Ørsted(9/99) model fitted a sample of independent $F$ data with an rms deviation of $7 \mathrm{nT}$, that value increased to $18 \mathrm{nT}$ when the same truncation was applied. Bearing in mind that at satellite altitude the crustal field is of the order of $5 \mathrm{nT}$, there was again no indication in these numerical results that there were any problems with the model.

It was slightly puzzling that, for all classes of data, the misfits for the 2000a model, were consistently very slightly 
less than those for the 2000c model. Individually the differences were not statistically significant (especially for the surface data), but the cumulative effect might just be significant. Despite this minor puzzle, we preferred to use the later model, because it used as much as possible of the Ørsted data then available.

Another reason for this choice of the later model was that, because of the limited amount of vector data used for the 2000a model, this earlier model showed a small Backus/ perpendicular-error effect, having equatorial maxima/ minima in $\delta Z$ of the order of $20 \mathrm{nT}$ for the full, parent, model (though only of magnitude about $5 \mathrm{nT}$ for the truncated $n \leq 10$ model). Another 1-month model (using 3 days in September) having a different data distribution, showed a similar effect, though by chance of roughly opposite sign. Fortunately the total vector data set was much more nearly uniformly distributed in the equatorial region, and we can expect any Backus effect in this 2000c model to be trivial.

However there still remained other puzzling features of the model. One was that the residuals between the parent model and the Ørsted data used in the modelling were not random, but showed a long-wavelength pattern. There was a strong suggestion in the residual field of some sort of a global $n=1, m=0, Y$ toroidal field of amplitude about 10 $\mathrm{nT}$, together with some sort of $n=1, m=0$ non-potential $X, Z$ field. (It was later realized that it is inherent in a least-squares fit that the residuals will be orthogonal to the model, in the sense that the weighted sum of (residual $\times$ model value) is zero; to the extent that this is true separately for the vector and scalar data, and that the vector data are uniformly distributed over the sphere, this implies that the 'field' defined by the vector residuals is orthogonal to the model field. As the model is a long-wavelength potential field we would therefore expect the residual field to be of short-wavelength and/or non-potential.)

When converted back to the local magnetometer coordinate system (which is rotating in space), these residuals showed a marked asymmetry, being smallest along the axis parallel to the axis of the star-camera. This was not too surprising, because of the better accuracy of pointing in this direction, but the magnitude of the asymmetry was larger than expected. The modellers also pointed out that when the part of the residual vector lying in the plane perpendicular to the star-camera axis is resolved into two components, one component ('N') perpendicular to the model field, and a third orthogonal component (' $\mathrm{B}$ '), it is the $\mathrm{N}$ component which is the larger. Again, to some extent this could be explained by noise in the star camera (and it was later realized that such an asymmetry would be also expected from the operation of the least square's process). So the split of the magnitude of the residual vector between the various components seemed not unreasonable; what was puzzling was its long-wavelength nature, perhaps suggesting that there were still problems with the relative orientation/timing of the star camera and magnetometer.

Another minor puzzle was with some of the correlations shown in the output covariance matrix. There were the expected fairly high negative correlations, of order 0.7 , between the respective constant and $D s t$-varying terms of the $n=1$ coefficients, indicating the difficulty of separating these two contributions when the data set includes only a small range of Dst. Similarly there were negative correlations, of order 0.25 , between the $g_{n}^{n}$ and $g_{n+2}^{n}$ coefficients (and similarly for $h$ ), suggesting the presence of a (small) Backus effect. But there were also positive correlations of order 0.25 between almost all of the $g_{n}^{n-1}$ and $h_{n}^{n}$ coefficients, with similar negative correlations between the $h_{n}^{n-1}$ and $g_{n}^{n}$ coefficients. (At the time the origin of these latter correlations was not known, but it has since been shown that they are just another expression of the Backus effect; this effect is linked to the geomagnetic axis, which is inclined with respect to the geographic axis on which the spherical harmonics are based.)

But these remaining problems had to be put into context. No previous modelling-process/candidate-model had been subject to such detailed scrutiny; for example it was not known whether the residuals produced in the modelling of the MAGSAT data produced similar problems. We knew the model was not perfect, but no model ever would be. We were satisfied that this candidate model IGRF2000c was fitting the independent observations we tested it against as well as could reasonably be expected, and was probably as accurate or better than previous IGRFs - we were thinking in terms of an accuracy of 30-50 nT.

Therefore on 11 November the Task Force formally accepted the Ørsted model (called above IGRF2000c) as the official IGRF 2000, and disbanded itself.

\section{A Personal View}

In retrospect I think we probably made a mistake in testing the truncated $n \leq 10$ model rather than the full parent model. When comparing competing candidate models against a given data set, it is clearly correct to test the $n \leq 10$ submissions. But our job was rather different, and we would probably have saved ourselves trouble and time if we had used the full $n \leq 13$ internal, static, model for the ground data, and the full parent model for the Ørsted $F$ data.

It is also now clear that if we had had to proceed to our original Stage 2 model, using only the $F$ data from Ørsted, our ground vector data-base would have had to have been considerably augmented in order to stabilize the model. We would almost certainly have had to also use synthetic "data" from a previous model, either as vectors, or as the position of the magnetic equator as was done by Mandea and Langlais (2000).

This particular Task Force certainly worked well in its specific task of testing a single candidate model for its acceptability. It has been suggested in the past that the Working Group decisions on accepting/averaging candidate models should be done away from the IAGA Assembly, by delegating the job to a similar group. Certainly, I have been the first to complain that the data available at the Working Group meeting was often inadequate to come to a properly informed decision! But I think there is a major difference between deciding if the only available model is acceptable, and between deciding between several competing models.

What has been improved already is that (most) candidate models are now accompanied by much more information about the data and method of analysis. Comparison of the candidate models against other, partial, data-bases is rarely very helpful in deciding on the relative weight to give to the 
candidate models, and in the past I suspect that decisions have often been influenced by (largely subjective) decisions on the quality of data-handling and modelling. (The 1999 situation was rather unusual in that the comparison with available databases showed how bad all of the candidate main-field models were!) Because of the large crustal "noise", the comparison with the independent surface data used by this Task Force would probably have shown up only gross inaccuracies in the model. It was the fact that we had full information about the data handling and analysis (and had contributed to the use of "best practice") that gave us confidence in our decision to accept.

So my present opinion is that the success of this particular Task Force does not necessarily mean that this is the way all future decisions should be taken. Many "man"-hours were involved, in this case justified partly because we felt we had a duty to the geomagnetic community to produce an acceptable IGRF 2000 on time. In the future there will perhaps be less urgency, and less willingness to divert effort away from more personal research.

As chairman of the Task Force, I cannot finish without taking this opportunity of thanking all those who participated, for their enthusiastic and thoughtful cooperation, often working against very tight time constraints; the geomagnetic community is indebted to them.

Acknowledgments. The author thanks the Goddard Space Flight Center for the invitation to visit in October 1999

\section{References}

Langel, R. A., R. H. Estes, and T. J. Sabaka, Uncertainty estimates in geomagnetic field modelling, J. Geophys. Res., 94, 12,281-12,299, 1989.

Langlais, B. and M. Mandea, An IGRF candidate main geomagnetic field model for epoch 2000 and a secular variation model for 2000-2005, Earth Planets Space, 52, this issue, 1137-1148, 2000.

Lowes, F. J., T. Bondar, V. P. Golovkov, B. Langlais, S. Macmillan, and M. Mandea, Evaluation of the candidate Main Field model for IGRF 2000 derived from preliminary Ørsted data, Earth Planets Space, 52, this issue, 1183-1186, 2000.

Macmillan, S., An evaluation of candidate geomagnetic field models for IGRF 2000, Earth Planets Space, 52, this issue, 1163-1165, 2000.

Mandea, M. and B. Langlais, Use of Ørsted scalar data in evaluating the pre-Ørsted main field candidate models for the IGRF 2000, Earth Planets Space, 52, this issue, 1167-1170, 2000.

Olsen, N., T. J. Sabaka, and L. Tøffner-Clausen, Determination of the IGRF 2000 model, Earth Planets Space, 52, this issue, 1175-1182, 2000.

Sabaka, T. J., R. A. Langel, R. T. Baldwin, and J. A. Conrad, The geomagnetic field 1900-1995, including the large-scale field from magnetospheric sources, and the NASA candidate models for the 1995 revision of the IGRF, J. Geomag. Geoelectr., 40, 157-206, 1997.

F. J. Lowes (e-mail: f.j.lowes@ncl.ac.uk) 